\title{
The Role of Computed Tomography in Pneumonia in Patients with Associated Coronavirus Infection
}

\author{
Jonibekov Jasur Jonibekovich \\ Bukhara State Medical Institute
}

\begin{abstract}
The appearance of COVID-19 has set tasks for healthcare professionals related to rapid diagnosis and provision of medical care to patients. The diagnosis of COVID-19 infection is based on the complex application of clinical, radiological and laboratory research methods[1].Radiation research methods used to diagnose patients with suspected COVID-19 and viral pneumonia of another nature include: radiography, computed tomography and ultrasound examination of the lungs. Radiation imaging data allow us to suspect lung damage of viral etiology (including COVID-19), influence the management of a particular patient, the treatment of complications or the formulation of an alternative diagnosis with a high probability of lung damage of viral etiology.
\end{abstract}

Detection of antibodies to SARS-CoV-2 is carried out using immunochemical methods. Class A (IgA) antibodies begin to form and are available for detection from about 2 days from the onset of the disease, reach a peak after 2 weeks and persist for a long time. Class M antibodies (IgM) begin to be detected approximately on the 7th day from the beginning of infection, reach a peak after a week and can persist for 2 months or more. From about the 3rd week or earlier, antibodies of class $\mathrm{G}$ ( $\operatorname{IgG}$ ) to SARS-CoV-2 are detected. A feature of the humoral response to infection is a small time interval between the appearance of IgM and IgG antibodies, and sometimes their simultaneous formation [8$10]$.

Studies have demonstrated the high efficiency of computed tomography (CT) of the chest organs in the diagnosis of pneumonia caused by SARS-CoV-2, and only in some studies this position was questioned[3,11,18]. However, most publications are devoted, in fact, to CT-semiotics of lung tissue damage, assessment of the prevalence of the process and the dynamics of its development. At the same time, the issues of the diagnostic significance of CT at the early stage of COVID-19, recognition and correct interpretation of lung damage in the first 5 days of the onset of symptoms of the disease remain insufficiently studied $[15,17]$. Based on the results of the analysis of data obtained during the study in patients with suspected SARS-CoV-2-pneumonia, it was demonstrated that CT is a highly sensitive method for diagnosing pathological changes in lung tissue.

According to the results of the conducted complex of clinical examination, the question of the type of medical care and the amount of additional examination is decided. The diagnosis is established on the basis of a clinical examination, epidemiological history and laboratory results, as well as radiation methods.

The purpose of the study. Study of the semiotics of pathological changes in the lungs according to CT data in the clinical symptoms of COVID-19.

Materials and methods of research. The study was based on the results of a comprehensive clinical, X-ray examination of the chest, computed tomography and PCR test, as well as ultrasound examination of the lungs in 112 patients. The patients were monitored from May 2020 to May 2021 in the X-ray room of the Bukhara Regional Infectious Diseases Hospital.All patients underwent 
computed tomography of the lungs using ToshibaAquilion 32 and GE revolution EVO 128 scanners. Tomography was performed in a routine mode at a voltage of $120 \mathrm{kV}$ on an X-ray tube. The program loaded a series of CT images of the chest with a slice thickness of $1 \mathrm{~mm}$.

When studying the period before the appearance of clinical symptoms of pneumonia caused by COVID-19 in patients, we obtained the following results: in 19 (16.9\%) patients - 1-7 days, in 72 patients-7-21 days (64.3\%) and in $21(18.7 \%)$ patients - 21 - 30 days. In patients under 40 years of age, the symptoms of COVID 19 pneumonia often ranged from 1 to 7 days, while in most patients of the older age group, the duration ranged from 7 to 21 days.

COVID-19 is usually manifested by fever in 91 patients $(81.3 \%)$, dry cough or with a small amount of sputum (66.0 and $61.6 \%$ ) and shortness of breath $(62.0 \%)$, other respiratory tract symptoms (runny nose, pain when swallowing $72.3 \%$ ), other signs of damage are possible (diarrhea and symptoms of gastrointestinal tract damage-20.5\%). Based on the data, we can say that the most common triad of symptoms was considered fever, dry cough and symptoms of gastrointestinal tract damage.

When determining the results of the PCR test, they were positive in 72 patients, i.e. $64.3 \%$, and negative results were in 39 cases, i.e. $34.8 \%$, in doubtful cases-in $1(0.9 \%)$ patient.

Risk factors for severe course include old age, diabetes mellitus, coronary heart disease, arterial hypertension and chronic obstructive pulmonary disease.

Based on the survey data of 112 patients with varying degrees of clinical and radiological picture and studied foreign publications on the current issue, approaches to the diagnosis of COVID-19 were determined.

In our study, the"frosted glass " symptom prevailed. This sign is represented by a small compaction of the parenchyma without its volumetric change, with partial preservation of pneumatization, due to which bronchial and vascular structures are observed. Numerous seals of the lung tissue according to the "frosted glass" type are mainly of a rounded shape, of various lengths with / without consolidation (Fig.1).
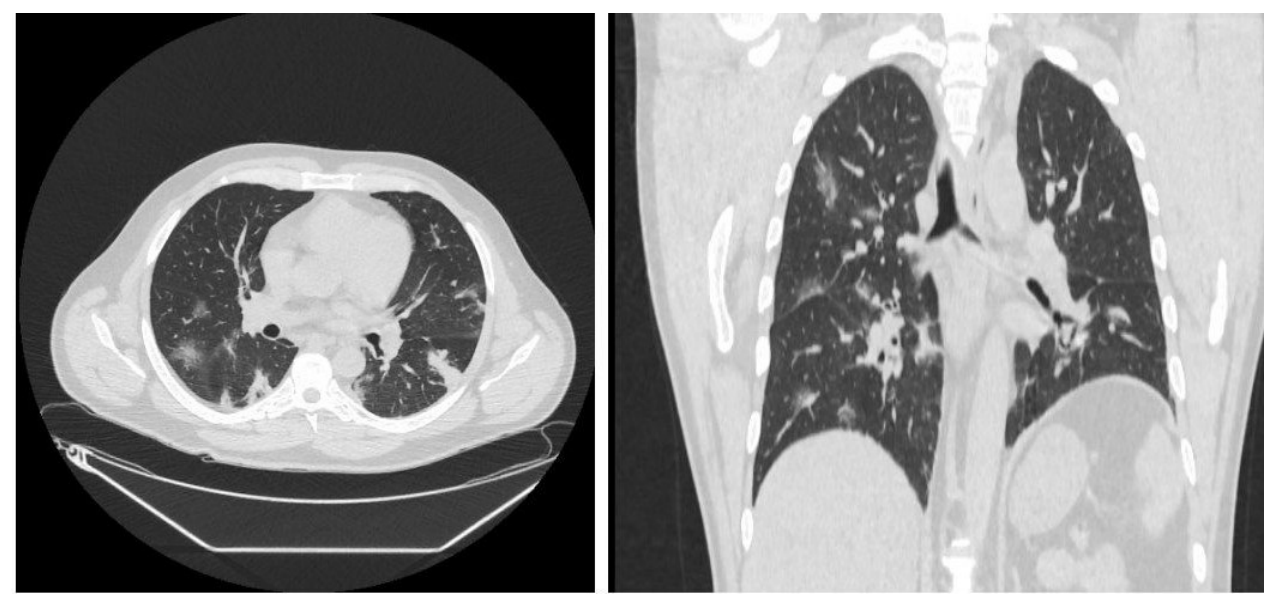

Fig. 1. Patient M., 46 years old. CT of the lungs, "pulmonary window", axial and coronary planes.

Areas of "frosted glass" with uneven and clear contours are defined along the periphery.

Most often, the "frosted glass" was bilateral - in 87 (79.0\%) patients, only subpleural lesions were detected in $23(20.9 \%)$, but there were patients in whom CT examination only revealed areas of "frosted glass" of both peripheral $15(13.6 \%)$ and central localization-9 (8.2\%). Among the typical manifestations of the "frosted glass" symptom, either drain foci were detected-in $74(67.3 \%)$ cases, or rounded foci - in $36(32.7 \%)$ cases. 
In $16(14.5 \%)$ cases, there was a lesion of the dorsal parts, in $94(85.4 \%)$ - a chaotic lesion of different segments, both peripheral and central localization (Fig. 2).

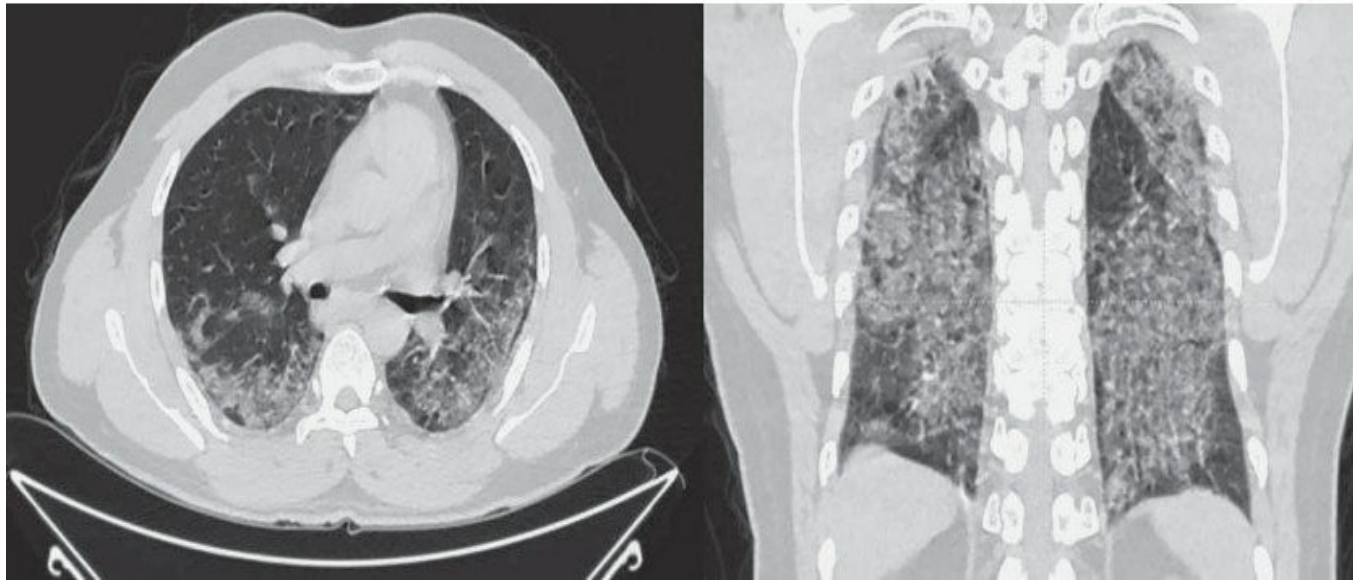

Fig. 3. Computed tomograms of the lungs. The patient is 49 years old. The dorsal parts of the lungs are more affected. The PCR test is negative.
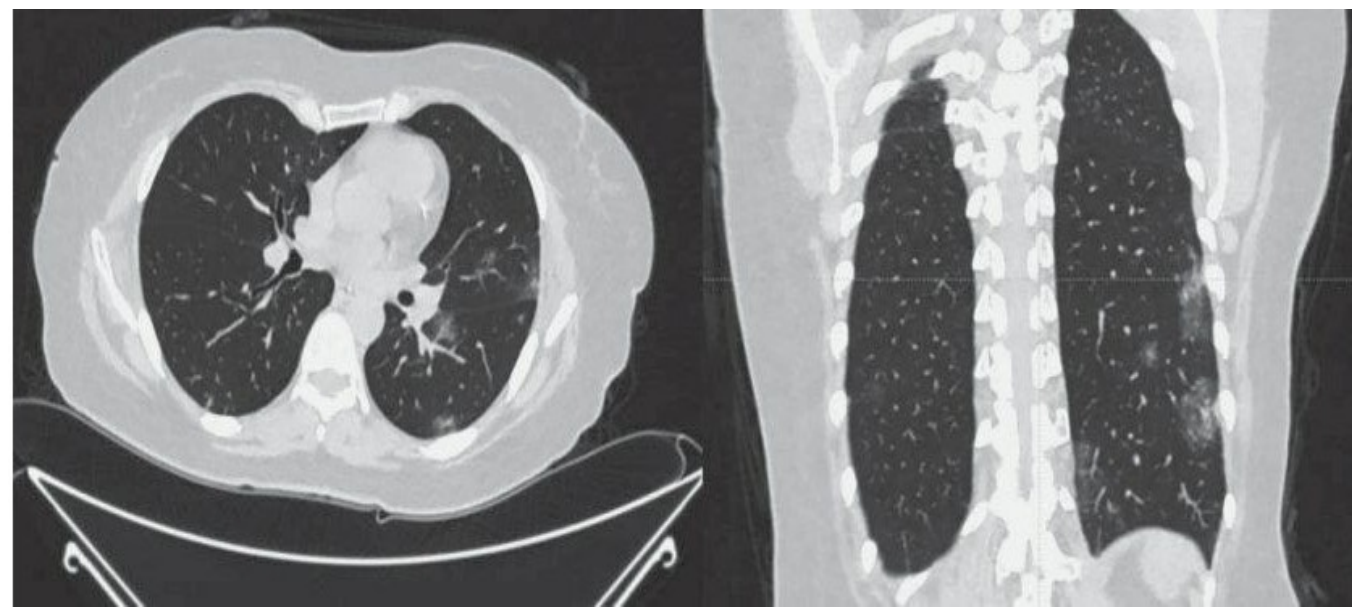

Fig. 4. Computed tomograms of the lungs. The patient is 55 years old. The dorsal parts of the lungs are more affected.

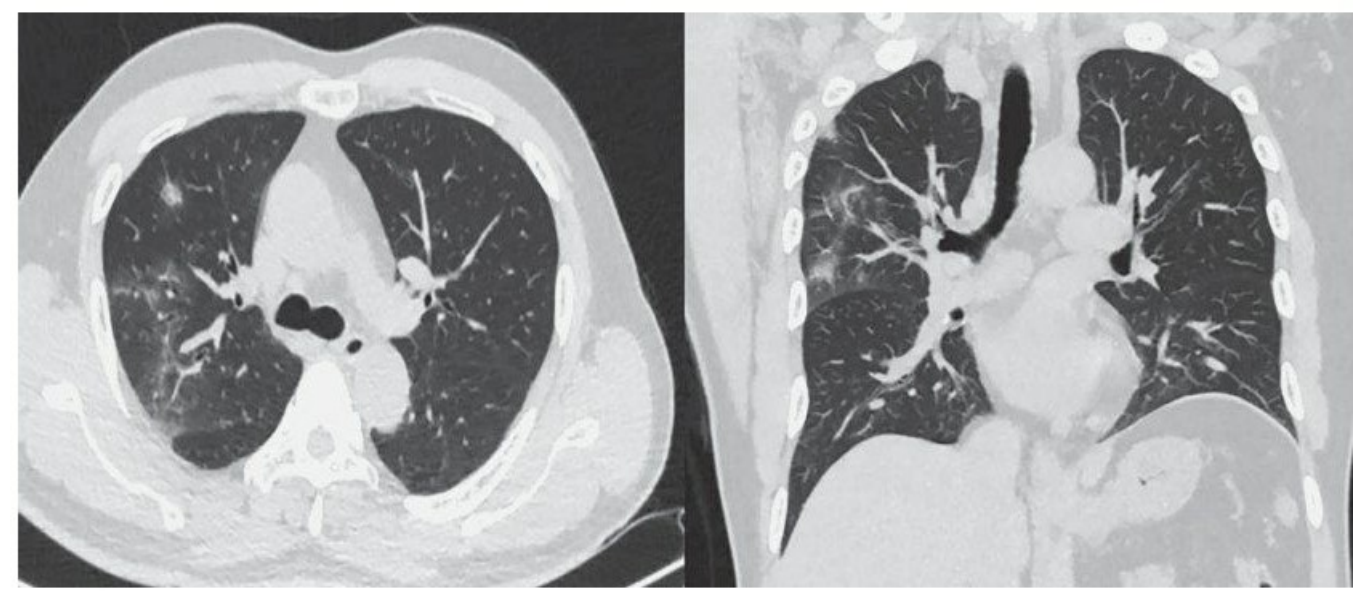

Fig. 5. Computed tomograms of the lungs. The patient is 45 years old. The dorsal parts of the lungs are more affected. 
The second most common symptom was the consolidation of lung tissue, it was observed in 54 patients (49.1\%). Consolidation on tomograms is visualized as a homogeneous compaction of the pulmonary parenchyma, which hides the walls of blood vessels and the walls of the respiratory tract

(Fig.5).

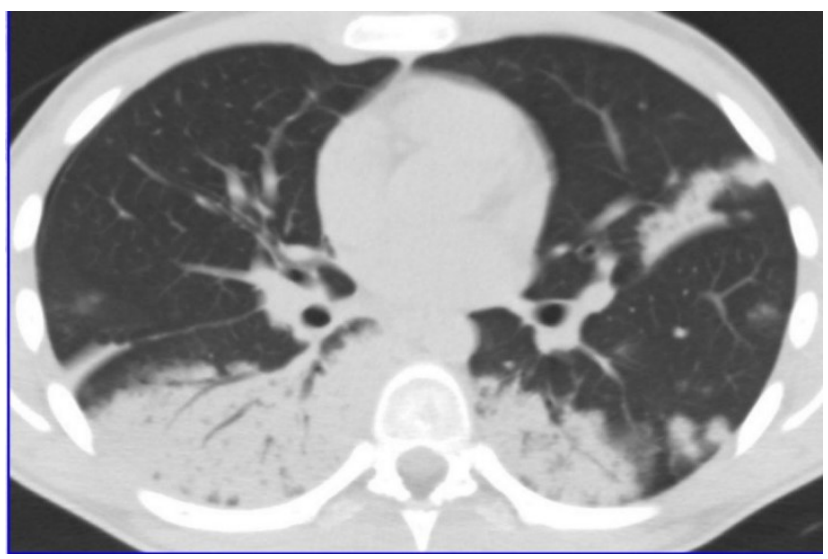

The different prevalence and semiotics of COVID-19-associated pneumonia indicate a different reaction of the body to infection. It is emphasized that it is necessary to comply with certain methodological requirements when performing MSCT in patients with pneumonia caused by SARSCoV-2. The effect of an air bronchogram against the background of consolidations in our patients has always been preserved. That is, the bronchi and bronchioles in COVID-19 are passable and are almost not involved in the process in the absence of bacterial infection.

It should be borne in mind that CT signs of COVID-19-associated pneumonia may lag behind or outstrip the clinical symptoms of the disease in terms of time of manifestation.

\section{Conclusions.}

1. Typical radiological manifestations of viral pneumonia (COVID-19) include -

$>$ a symptom of the "frosted glass" type, numerous compressions of the lung tissue of mainly rounded shape, of various lengths with / without consolidation, peripheral, multilobar localization; - the lesion is more often bilateral in nature;

$>$ thickening of the interlobular interstitial according to the type of "cobblestone pavement";

$>$ areas of consolidation, perilobular seals; - a symptom of an air bronchogram, traction bronchiectasis;

$>$ pleural effusion, hydrothorax (bilateral, predominant on the left).

2. The percentage of damage is estimated separately for each lung. The degree of changes is determined by the lung with the greatest lesion.

3. Computed tomography of the chest organs is recommended for use: in outpatient conditions in patients with suspected COVID-19 (including after radiography of the OGC); CT imaging is indicated for medical sorting of patients with suspected COVID-19 who have clinical signs of moderate severity and its high probability before receiving test results. CT imaging is not indicated for patients with suspected COVID-19 and moderate clinical signs, without risk factors for disease progression.

Given the high contagiousness of this infection and the constant need for radiation research methods, it is necessary to protect medical workers as much as possible. In patients with a mild or moderate course, with positive dynamics, complete lung recovery can be observed. In patients with a severe 
course, negative dynamics may be observed with the progression of the disease: as an increase in the prevalence of interstitial changes, the transformation of" frosted glass " into gross reticular changes or into massive areas of consolidation of lung tissue. CT of the chest organs, taking into account its high sensitivity, helps in making a diagnosis, monitoring the course of the disease, playing an important role in making clinical decisions in the treatment of COVID-19.

\section{List of Literature}

1. Chung M., Bernheim A., Mei X., Zhang N., Huang M., Zeng X. et al. CT Imaging Features of 2019 Novel Coronavirus (2019-nCoV). Radiology. 2020; 295: 202-7.

2. Inui S., Fujikawa A., Jitsu M., et al. Chest CT Findings in Cases from the Cruise Ship "Diamond Princess" with Coronavirus Disease 2019 (COVID-19). Radiology: Cardiothoracic Imaging. 2020; 2 (2). Published Online.

3. Fang Y., Zhang H., Xie J., Lin M., Ying L., Panget P. et al. Sensitivity of Chest CT for COVID19: Comparison to RT-PCR [published online ahead of print, 2020 Feb 19]. Radiology. 2020.

4. Franquet T. Imaging of pulmonary viral pneumonia. Radiology. 2011; 260 (1): 18-39.

5. Kligerman S.J., Franks T.J., Galvin J.R. From the radiologic pathology archives: organization and fibrosis as a response to lung injury in diffuse alveolar damage, organizing pneumonia, and acute fibrinous and organizing pneumonia. Radiographics. 2013; 33 (7): 1951-75.

6. Rubin G.D., Ryerson C.J., Haramati L.B., Sverzellati N., Kanne J. P., Raoof S. et al. The Role of Chest Imaging in Patient Management during the COVID-19 Pandemic: A Multinational Consensus Statement from the Fleischner Society Radiology. 2020;

7. Revel M.P., Parkar A.P., Prosch H., Silva M., Sverzellati N., Gleeson F. et al. COVID-19 patients and the Radiology department - advice from the European Society of Radiology (ESR) and the European Society of Thoracic Imaging (ESTI). Eur. Radiol. 2020.

8. Pan F., Ye T., Sun P., Guil Sh., Liang B., Li L. et al. Time Course of Lung Changes On Chest CT During Recovery From 2019 Novel Coronavirus (COVID-19) Pneumonia .Radiology. 2020.

9. Soldati G., Smargiassi A., Inchingolo R. Buonsenso D., Perrone T., Briganti D.F. et al. Proposal for International Standardization of the Use of Lung Ultrasound for Patients With COVID-19: A Simple, Quantitative, Reproducible Method. J. Ultrasound Med. 2020;

10. Piscaglia F., Stefanini F., Cantisani V., Sidhu P.S., Barr R., Berzigotti A. et al. Benefits, Open questions and Challenges of the use of Ultrasound in the COVID-19 pandemic era. The views of a panel of worldwide international experts .Ultraschall Med. 2020.

11. Lu W., Zhang Sh., Chen B., Chen J., Xian J., Lin Yu. et al. A Clinical Study of Noninvasive Assessment of Lung Lesions in Patients with Coronavirus Disease-19 (COVID-19) by Bedside Ultrasound. Ultraschall Med. 2020. 